\title{
The clinical consequence of positive mental health in psychotherapy
}

\author{
Mario Fulcheri, ${ }^{1}$ Danilo Carrozzino ${ }^{1,2}$ \\ ${ }^{1}$ Department of Psychological, Health, and Territorial Sciences, University G. d'Annunzio of Chieti-Pescara, Chieti, Italy; ${ }^{2}$ Psychiatric \\ Research Unit, Psychiatric Centre North Zealand, Copenhagen University Hospital, Hillerød, Denmark
}

\begin{abstract}
The current commentary is aimed at critically analyzing the document Psychotherapies for Anxiety and Depression: benefits and costs by focusing on specific theoretical concepts and empirical evidences arising from research studies fulfilled in the area of study of Clinical and Health Psychology. Specifically, the following were the main topics on which we are focused on: i) the clinical consequence potentially resulting from considering the psychological well-being and the euthymia condition as the main targets of a psychotherapeutic treatment; ii) a critical reappraisal of the clinical inadequacy of the evidence based model in psychotherapy; iii) clinimetrics as a clinically based measurement method for evaluating the psychological well-being of a patient after a psychotherapeutic intervention.
\end{abstract}

Key words: Clinimetrics; Euthymia; Positive psychology; Psychological well-being; Well-being therapy.

\section{Commentary}

The document Psychotherapies for Anxiety and Depression: benefits and costs addresses a clinically relevant research topic by providing a critical overview on psychological interventions for the treatment of anxiety and depressive symptoms. This document was presented for the first time during a Conference in November 2016, in Padua, Italy. Whereas the objective of such contribution

Correspondence: Danilo Carrozzino, Department of Psychological, Health, and Territorial Sciences, University "G. d'Annunzio" of Chieti-Pescara, Via dei Vestini no. 31, 66100 Chieti, Italy.

Tel: +393343204782 .

E-mail: danilo.carrozzino@unich.it

Contributions: both authors equally participated in the conceptualization and writing of this manuscript by approving its final version.

Conflict of interest: the authors declare no potential conflict of interest.

Citation: Fulcheri, M., \& Carrozzino, D. (2017). The clinical consequence of positive mental health in psychotherapy. Research in Psychotherapy: Psychopathology, Process and Outcome, 20(2), 143-146. doi: 10.4081/ripppo.2017.280

Received for publication: 21 May 2017.

Revision received: 18 June 2017.

Accepted for publication: 20 June 2017.

This work is licensed under a Creative Commons Attribution NonCommercial 4.0 License (CC BY-NC 4.0).

(C) Copyright M. Fulcheri and D. Carrozzino, 2017

Licensee PAGEPress, Italy

Research in Psychotherapy:

Psychopathology, Process and Outcome 2017; 20:143-146

doi:10.4081/ripppo.2017.280 was mainly focused on applying the evidence based principles to psychological treatments, the current commentary is aimed at critically analyzing this widely debated research topic according to the scientific viewpoint of the Clinical and Health Psychology (Bertini et al., 2014). Searching for a link between Clinical and Health Psychology (Bertini et al., 2014) potentially means expanding the traditional concept of disease by moving from the classical medical diagnosis (i.e., identifying mere somatic or organic symptoms) toward a more comprehensive evaluation of the illness behavior (Mechanic \& Volkart, 1960). The concept of illness behavior comprises the varying ways to perceive, evaluate and to respond to symptoms at the individual level (Mechanic \& Volkart, 1960). This individual domain operates as unifying factor encompassing the several biological (e.g., physical dimensions), psychological (e.g., personality traits, emotional components), and social (e.g., cultural elements) variables (Morin, 1992) affecting the subjective health status (Mechanic \& Volkart, 1960).

On this preliminary theoretical background, when taking the high prevalence rates of anxiety and depression symptoms in the general population into consideration, a large body of literature extensively highlighted the clinical utility of improving the access to psychological treatments, including psychotherapeutic interventions (Dodd, Joyce, Nixon, Jennison, \& Heneage, 2011). In order to fulfill this aim, we hereby underlined the potential clinical relevance of specific factors acting as theoretical concepts, methodological aspects, and scientific evidences that we evaluate as useful to further extend the actual applicability of psychotherapy within a National Health Care System. Specifically, we aimed to point out that promoting, during the clinical process of a psychotherapy, a positive mental health condition among people from general population presenting with sub-threshold, acute or 
residual symptoms of anxiety or depression should not only implicate removing symptoms by pathologizing and medicalizing all the health complaints of the patient (Fava, Fabbri, \& Sonino, 2002). By constrast, when focusing on the World Health Organization (WHO) criteria for both determining the health status and identifying the levels of recovery of a patient after a psychological treatment, it is clinically relevant not only treating psychopathological symptoms but also instilling a positive mental state by focusing on an attainment of specific psychological factors (e.g., well-being, resilience, coping). The attainment of these positive psychological factors has been associated with significantly diminished risk of recurrence of depression and anxiety disorders, as well as with significantly lower rates of relapse (Fava, Cosci, Guidi, \& Tomba, 2017). Although the etymology of the term clinical psychology with its underlying meaning of medical practice at the sickbed still significantly influences the clinical intervention of many psychologists and psychotherapists emphasizing the importance of alleviating the mental illness rather than facilitating positive psychological factors leading to mental health, a positive clinical psychological approach has been conceived a very relevant issue for deconstructing an illness ideology and constructing an ideology of human strengths (Maddux, Snyder, \& Lopez, 2004).

Such a clinical approach, including a positive psychological perspective, is in line with the Adlerian concept of encouragement as psychotherapeutic strategy aimed at achieving a mental health condition not only by focusing on manifest symptoms but also by promoting a psychological well-being (Watts \& Pietrzak, 2000). This intervention of encouragement should not to be interpreted as the core curing process of a psychotherapy but as a specific technique emphasizing the clinical utility of promoting positive beliefs and behaviors for facilitating an optimal individual functioning (Watts \& Pietrzak, 2000). Indeed, regardless of the specific theoretical orientation (e.g., cognitive-behavioral, psychodynamic), the encouragement process is only one of many psychotherapeutic strategies that the clinician may select and integrate with other useful clinical approaches for enhancing the patient's well-being (Watts \& Pietrzak, 2000).

Thus, although psychoanalysis with its focus on hidden intrapsychic processes provided a fertile soil for the development of a prevalent illness ideology (Maddux et al., 2004), this clinical positive viewpoint historically derives from psychodynamic psychotherapy with first psychoanalytic research studies testing novel psychotherapeutic strategies for handling psychological resistance by facilitating a positive psychological functioning (Strean, 1985). However, despite the initial focus on such a positive psychological ideology, the subsequent development of several psychotherapeutic approaches was progressively mainly focused on clinical strategies leading to symptom reduction rather than to a well-being attain- ment (Parloff, Kelman, \& Frank, 1954). Indeed, the achievement of such positive psychological factors during a psychotherapy was considered only as a secondary consequence (i.e., a type of by-product) of the reduction of symptoms (Parloff, Kelman, \& Frank, 1954). Nowadays, we have a lot of empirical data evidencing that equating the presence of a mental health condition with the mere absence of psychopathological symptoms consists of a diagnostically inadequate method and clinically reductionistic viewpoint (Fava et al., 2017). As argued also by Jahoda (1958) who stated that the absence of symptoms may constitute a necessary, but clinically not sufficient factor for identifying a mental health condition and then further sustained by Ryff (2014) with her comprehensive model of psychological well-being, the traditional targets and goals of a psychotherapeutic treatment (e.g., full remission of symptoms) should be integrated with the pursuit of positive psychological outcomes such as an optimistic evaluation of one's self, an individual sense of continued growth, the belief that life is purposeful and meaningful, the possession of quality relations with others, the ability to face daily problems with self-determination and psychological flexibility (Fava et al., 2017). As reported also in the previous statements, the clinical relevance of these psychological variables derives from a lot of empirical evidences showing that the development of such positive mental factors has been associated with significantly lower rates of relapse after a depression or during the residual phase of an anxiety disorder (Fava et al., 2017; Hardeveld, Spijker, De Graaf, Nolen, \& Beekman, 2010). This emerging clinical perspective has recently seen further developments with the potentially innovative concept of euthymia (Bech, Carrozzino, Austin, Møller, $\&$ Vassend, 2016; Fava \& Bech, 2016). The euthymic condition encompasses several positive psychological aspects (e.g., lack of mood disorders, feeling calm, cheerful and active, refreshing or restorative sleeping, as well as resistance to stress and frustration tolerance) (Fava \& Bech, 2016). The clinical advantage potentially arising from including the euthymic state among the therapeutic outcomes of a psychological treatment may consist of promoting an individualized process of rehabilitation focused on a biopsychosocial and personalized recovery model from mood disorders, as well as from anxiety disturbances (Fava et al., 2017). This comprehensive recovery model is clinically related to the evidence of roll-back phenomenon provided by Detre and Jarecki (1971) who showed a specific temporal relationship between the prodromal and the residual symptoms of a psychiatric disorder. Specifically, they (Detre \& Jarecki, 1971) have found that when the symptoms of a psychiatric disorder remitted, there is a clinically significant risk that the residual symptomatology progressively tends to develop in reverse order as prodromal symptoms of relapse (Fava et al., 2002). This clinical evidence was the basis for the development of the sequential model for the treatment of depression as origi- 
nally introduced by Fava (1999) who combined the use of pharmacotherapy for the acute depressive episode with a specific psychotherapeutic intervention addressed to the residual phase of depression. Such a clinical intervention, incorporating in the definition of recovery the psychological well-being as the main target of the treatment, was further validated with the development of a specific psychotherapeutic strategy for enhancing an optimal functioning of the patient (e.g., environmental mastery, personal growth, purpose in life, autonomy, self-acceptance, positive relations with others) (Fava et al., 2017). This shortterm psychotherapeutic technique, defined as Well-Being Therapy (WBT), consists of a psychological treatment specifically manualized and elaborated not only for increasing the levels of psychological well-being of the patient but also for promoting a specific euthymic condition (Fava et al., 2017). The WBT was extensively validated according to the evidence-based guidelines in a number of independent randomized controlled trials (RCT) indicating its efficacy and effectiveness (Fava et al., 2017). However, applying the evidence based model in psychotherapy has been considered a controversial issue due to its potentially misleading method providing an oversimplification of treatment efficacy when neglecting the multiple and bidirectional interactions between the specific (e.g., the different psychotherapeutic strategies deriving from the specific conceptual framework) and nonspecific ingredients (e.g., the working alliance referring to qualities of the therapeutic relationship due to the degree of warmth, empathy, acceptance and respect between the therapist and the patient) affecting the clinical outcomes of a psychotherapeutic intervention (Butler \& Strupp, 1986; Fava, Guidi, Rafanelli, \& Sonino, 2015). Indeed, when strictly focusing on RCT data according to the evidence based guidelines, results may indicate the comparative efficacy of diverse psychotherapeutic treatments for the average randomized patient but not for those whose individual symptoms, including the severity of the clinical condition and the potential comorbidities, deviate from standard presentations (Fava et al., 2015). The major prognostic risk here comprises a significantly increased likelihood to neglect the patient's individual perception of own health in response to a specific psychological treatment. For instance, although the efficacy of antidepressant drugs is widely acknowledged in the treatment of major depression, the individual response to this pharmacological intervention may vary from patient to patient due to the specific severity of symptoms (e.g., mild, moderate, severe) or because of the different staging of the disorder as well as for the several adverse effects potentially occurring during the therapy (Fava et al., 2015). Similarly, when validating a psychotherapeutic intervention according to RCT guidelines that are likely to neglect the individual responsiveness, it is highly relevant from a clinical point of view accounting for the psychological well-being individually perceived by the patient (Fava et al., 2015). Thus, the aim of a clinical trial should not only consist of demonstrating the statistical superiority of a psychological treatment compared to a control therapy as psychometrically evaluated in a short-term study. Conversely, it is highly relevant from a clinical point of view longitudinally examining the complex subjective responsiveness and vulnerability to the specific psychotherapeutic intervention according to a more comprehensive conceptual framework linking the clinical judgment of the experienced psychotherapist to the individual well-being selfreported by the patient (Fava et al., 2015). Clinimetrics, defined as measurement based care focusing on clinically valid self-rating scales and questionnaires to assess the balance between the desired clinical outcomes of a treatment and the undesirable side effects, may provide a useful solution in this regard by evaluating to what extent the patient has returned to his or her usual state of psychological well-being (Bech, 2016). Specifically, when considering that the efficacy of the evidence based medicine has not yet been tested in comparison to the experienced clinical judgment, one of the main objectives of the clinimetric approach consists of increasing the dialogue between the patient and the psychotherapist within a collaborative setting where the patient's well-being is considered the core outcome to achieve with a specific psychological treatment (Bech, 2016). Therefore, clinimetrics is aimed at providing a comprehensive assessment of clinical outcomes of a psychotherapeutic intervention by analyzing the following relevant aspects: i) wanted and expected treatment effects (e.g., the potential clinical benefits); ii) treatment-induced unwanted or adverse side effects (e.g., behavioral toxicity, iatrogenic comorbidity); iii) the patient's own individual experience of a change in terms of well-being or euthymic condition (Fava, Tomba, \& Bech, 2017). Specific rating-scales were recently clinimetrically analyzed and recommended as clinically valid instruments for detecting the clinical benefits of a psychotherapeutic intervention, as well as the individual responsiveness to such a psychological treatment, including the potential adverse effects that may occur during the therapy (Carrozzino et al., 2016; Tomba \& Bech, 2012). More specifically, whereas rating-scales (e.g., the Derogatis SCL-90-R), only including negatively worded items, are clinimetrically indicated for measuring psychopathological symptoms (Carrozzino et al., 2016), scales exclusively containing positively formulated items as the WHO-5 Well-Being Index are recommended for capturing improvement in well-being due to a specific psychotherapeutic intervention (Topp, Østergaard, Søndergaard, \& Bech, 2015). Similarly, the Shedler-Westen Assessment Procedure (SWAP-200), integrating, according to the aforementioned clinimetric principles, the global evaluations of the experienced clinician with specific statistical analyses, is a clinically valid assessment tool for detecting personality change in psychotherapy (Lingiardi, Shedler, \& Gazzillo, 2006). 


\section{Conclusions}

Finally, as main future research directions and clinical perspectives resulting from our positively formulated psychological issues, we hope for a promising role of University residency programs in Clinical and Health Psychology for further promoting within the National Health Care System clinically suitable public psychological services aimed at reappraising the positive mental health as the main target of a psychotherapeutic intervention. More specifically, concerning future studies, the major aim consists of providing new research insights on the clinical link between positive psychology and psychotherapy by further evaluating the consequence of wellbeing and euthymia on psychotherapeutic outcomes. Thus, in terms of implications for the clinical practice, it is time to clinimetrically implement the relevance of positive clinical psychology for psychotherapy.

\section{References}

Bech, P. (2016). Measurement-based care in mental disorders. New York: Springer.

Bech, P., Carrozzino, D., Austin, S.F., Møller, S.B., \& Vassend, O. (2016). Measuring euthymia within the Neuroticism Scale from the NEO Personality Inventory. A Mokken analysis of the Norwegian general population study for scalability. Journal of Affective Disorders, 193, 99-102. doi: http:// dx.doi.org/10.1016/j.jad.2015.12.039

Bertini, M., Freda, M.F., Fulcheri, M., Carrozzino, D., Molinari, E., \& Solano, E. (2014). Commenti all'articolo "Pratiche di salute, pratiche di psicologia: per una professionalizzazione della Psicologia della Salute in Italia" (Family of rules and family of affects. Which are the consequences on the wellbeing of adolescent offsprings?). Psicologia della Salute, 2, 26-55. doi: 10.3280/PDS2014-002003

Butler, S.F., \& Strupp, H.H. (1986). Specific and nonspecific factors in psychotherapy: A problematic paradigm for psychotherapy research. Psychotherapy: Theory, Research, Practice, Training, 23, 30-40. doi: http://dx.doi.org/10.1037/h0085590

Carrozzino, D., Vassend, O., Bjørndal, F., Pignolo, C., Raabæk, L., Bech, P. (2016). A clinimetric analysis of the Hopkins Symptom Checklist (SCL-90-R) in general population studies (Denmark, Norway, and Italy). Nordic Journal of Psychiatry, 70, 374-379. doi: http://dx.doi.org/10.3109/ 08039488.2016.1155235

Detre, T.P., \& Jarecki, H. (1971). Modern psychiatric treatment. Philadelphia, PA: Lippincott.

Dodd, K., Joyce, T., Nixon, J., Jennison, J., \& Heneage, C. (2011). Improving access to psychological therapies (IAPT): are they applicable to people with intellectual disabilities? Advances in Mental Health and Intellectual Disabilities, 5(2), 29-34. doi: http://dx.doi.org/10.5042/amhid.2011.0110

Fava, G.A. (1999). Sequential treatment. Psychotherapy and Psychosomatics, 68, 227-229. doi: https://doi.org/10.1159/ 000012338

Fava, G.A., \& Bech, P. (2016). The concept of euthymia. Psychotherapy and Psychosomatics, 85, 1-5. doi: 10.1159/ 000441244
Fava, G.A., Cosci, F., Guidi, J., \& Tomba, E. (2017). Well-being therapy in depression: new insights into the role of psychological well-being in the clinical process. Depression and Anxiety, 00, 1-12. doi: https://doi.org/10.1002/da.22629

Fava, G.A., Fabbri, S., \& Sonino, N. (2002). Residual symptoms in depression: an emerging therapeutic target. Progress in Neuro-Psychopharmacology \& Biological Psychiatry, 26(6), 1019-1027. doi: https://doi.org/10.1016/S0278-5846 (02)00226-9

Fava, G.A., Guidi, J., Rafanelli, C., \& Sonino, N. (2015). The clinical inadequacy of evidence-based medicine and the need for a conceptual framework based on clinical judgment. Psychotherapy and Psychosomatics, 84, 1-3. doi: 10.1159/000366041

Fava, G.A., Tomba, E., Bech, P. (2017). Clinical pharmacopsychology: conceptual foundations and emerging tasks. Psychotherapy and Psychosomatics, 86, 134-140. doi: 10.1159 /000458458

Hardeveld, F., Spijker, J., De Graaf, R., Nolen, W.A., \& Beekman, A.T. (2010). Prevalence and predictors of recurrence of major depressive disorder in the adult population. Acta Psychiatrica Scandinavica, 122(3), 184-191. doi: 10.1111/ j.1600-0447.2009.01519

Jahoda, M. (1958). Current Concepts of Positive Mental Health. New York, NY: Basic Books.

Lingiardi, V., Shedler, J., \& Gazzillo, F. (2006). Assessing personality change in psychotherapy with the SWAP-200: a case study. Journal of Personality Assessment, 86, 23-32. doi: http://dx.doi.org/10.1207/s15327752jpa8601_04

Maddux, J.E., Snyder, C.R., \& Lopez, S.J. (2004). Toward a positive clinical psychology: deconstructing the illness ideology and constructing an ideology of human strengths and potential. In P.A. Linley \& Joseph S. (Eds.), Positive Psychology in practice (pp. 320-334). Hoboken, NJ, US: John Wiley \& Sons.

Mechanic, D., \& Volkart, E.H. (1960). Illness behavior and medical diagnoses. Journal of Health and Human Behavior, I(2), 86-94.

Morin, E. (1992). From the concept of system to the paradigm of complexity. Journal of Social and Evolutionary Systems, 15(4), 371-385. doi: https://doi.org/10.1016/1061-7361(92) 90024-8

Parloff, M.B., Kelman, H.C., \& Frank, J.D. (1954). Comfort, effectiveness, and self-awareness as criteria of improvement in psychotherapy. American Journal of Psychiatry, 11, 343-351.

Ryff, C.D. (2014). Psychological well-being revisited: advances in the science and practice of eudaimonia. Psychotherapy and Psychosomatics, 83, 10-28. doi: https://doi.org/10.1159 1000353263

Strean, H.S. (1985). Resolving resistances in psychotherapy. New York, NY: Wiley.

Tomba, E., \& Bech, P. (2012). Clinimetrics and clinical psychometrics: macro- and micro-analysis. Psychotherapy and Psychosomatics, 81, 333-343. doi: https://doi.org/10.1159/ 000341757

Topp, C.W., Østergaard, S.D., Søndergaard, S., \& Bech. P. (2015). The WHO-5 well-being index: a systematic review of the literature. Psychotherapy and Psychosomatics, 84, 167-176. doi: 10.1159/000376585

Watts, R.E., \& Pietrzak, D. (2000). Adlerian "encouragement" and the therapeutic process of solution-focused brief therapy. Journal of Counseling \& Development, 78, 442-447. doi: 10.1002/j.1556-6676.2000.tb01927.x 